\title{
Antiferromagnetic domain size and exchange bias
}

\author{
M. R. Fitzsimmons, ${ }^{1}$ D. Lederman, ${ }^{2}$ M. Cheon, ${ }^{2}$ H. Shi, ${ }^{2, *}$ J. Olamit, ${ }^{1}$ Igor V. Roshchin, ${ }^{3,4}$ and Ivan K. Schuller ${ }^{3}$ \\ ${ }^{1}$ Los Alamos National Laboratory, Los Alamos, New Mexico 87545, USA \\ ${ }^{2}$ Department of Physics, West Virginia University, Morgantown, West Virginia 26506, USA \\ ${ }^{3}$ Department of Physics, University of California at San Diego, La Jolla, California 92093, USA \\ ${ }^{4}$ Department of Physics, Texas A\&M University, College Station, Texas 77843, USA
}

(Received 11 April 2008; published 2 June 2008)

\begin{abstract}
Using neutron diffraction, we measured the sizes of antiferromagnetic domains in three ferromagnet/ antiferromagnet bilayer samples as a function of the magnitude and sign of exchange bias, temperature, and antiferromagnet composition. Neutron-scattering techniques were applied to thin films with masses less than $10 \mu \mathrm{g}$. We found the antiferromagnetic domain size to be consistently small regardless of the exchange bias. For a Co/untwinned single crystalline antiferromagnet (AF)-fluoride bilayer, the antiferromagnetic domain size is comparable to the crystallographic domain size of the AF. For one sample the highest temperature at which the exchange bias was nonzero (i.e., the blocking temperature) was suppressed by $\sim 3 \mathrm{~K}$ compared to the Néel temperature of the antiferromagnet.
\end{abstract}

DOI: 10.1103/PhysRevB.77.224406

PACS number(s): 75.70.Ak, 75.70.Cn, 61.05.F-

\section{INTRODUCTION}

Exchange bias, the shift $H_{E}$ of the magnetization hysteresis loop of a ferromagnet (FM) about the applied field of zero, is commonly observed when unpinned spins of a ferromagnet are coupled to pinned spins of an antiferromagnet (AF). This coupling inhibits the response of unpinned spins to the applied field. In the absence of field, the coupling may cause the unpinned spins to point in one particular direction.

Exchange bias was originally reported by Meiklejohn and $\mathrm{Bean}^{1,2}$ in 1956 for $\mathrm{Co} / \mathrm{CoO}$ microparticle systems and subsequently in many other systems. ${ }^{3,4}$ Exchange bias has important technological applications in "spin-valve" devices, ${ }^{5}$ such as giant magnetoresistive read heads and magnetic random access memory. Such devices are based on a switch that can be fabricated from two FM layers-one pinned and the other unpinned. When the magnetic moment of the unpinned layer changes relative to the pinned layer, a change in resistance across the layers is produced.

Most theories of exchange bias require uncompensated spins in the AF that inhibit the response of a proximal FM. ${ }^{6,7}$ For example, the original direct coupling mechanism proposed by Meiklejohn and Bean, ${ }^{1,2}$ the local interface random exchange model proposed by Malozemoff, ${ }^{8}$ the AF domain wall formation mechanism proposed by Mauri et al., ${ }^{9}$ and the spin-flop mechanism combined with interface defects proposed by Schulthess and Butler ${ }^{10}$ all rely on a net uncompensated magnetization (even if it is local) of the AF at the $\mathrm{FM} / \mathrm{AF}$ interface. In many models, $\left|H_{E}\right|$ is proportional to the magnitude of the uncompensated magnetization at the FM/AF interface, and this magnitude scales inversely with the size of the region over which the magnetization is averaged. ${ }^{8,10}$ One question is whether the region over which the averaging takes place is the AF domain or the domain of uncompensated magnetization in the AF. By AF domain, we mean the region that exhibits long range order of the AF spin structure. The AF domain may not be the same as the domain of uncompensated magnetization in the AF. Furthermore, the AF domain in the film bulk may not be the same as the AF domain near the FM/AF interface.

One way to experimentally control the uncompensated magnetization is to use dilute antiferromagnets, in which do- mains form with a net uncompensated magnetization at the domain boundaries or inside the domains due to missing magnetic neighbors. ${ }^{11,12}$ Factors of 2 or greater enhancements of $H_{E}$ for dilute $\mathrm{FM} / \mathrm{AF} \mathrm{Co}_{x} \mathrm{Mg}_{1-x} \mathrm{O} / \mathrm{Co}$ bilayers were found for $x \sim 0.80$ (compared to $x=1$ ), ${ }^{11-13}$ and more recently enhancements of $H_{E}$ in polycrystalline $\mathrm{Co}_{x} \mathrm{Mg}_{1-x} \mathrm{O} / \mathrm{Co}$ bilayers have been observed. ${ }^{14} \mathrm{In} \mathrm{Co} /$ twinned $\mathrm{Zn}_{0.17} \mathrm{Fe}_{0.83} \mathrm{~F}_{2}$ bilayers, a $65 \%$ increase in $H_{E}$ with respect to pure $\mathrm{Co} /$ twinned $\mathrm{FeF}_{2}$ bilayers was observed. ${ }^{15}$ However, in Co/untwinned single crystalline $\mathrm{Fe}_{x} \mathrm{Zn}_{1-x} \mathrm{~F}_{2}$ bilayers, no significant enhancement of $H_{E}$ was observed, presumably due to a lack of percolation of nonmagnetic impurities at higher $\mathrm{Fe}$ concentrations. ${ }^{16}$

Another mechanism that leads to a net FM/AF coupling is the unequal coupling of the ferromagnet's spins to the two sublattices of the AF. In transition metal fluorides, unequal coupling could be a consequence of the different symmetries of fluoride ions for the two sublattices. ${ }^{17}$ (The different symmetries may also be a reason for piezomagnetism in these materials.) Alternative mechanisms that explain enhanced coercivity in exchange coupled bilayers and net magnetization in AF films attribute these phenomena to spin fluctuations on the AF surface. ${ }^{18,19}$

Uncompensated spins in the antiferromagnet film bulk can affect exchange bias. For example, the combination of a net magnetization in a nominally antiferromagnetic material coupled with antiferromagnetic exchange coupling at the FM/AF interface can lead to a positive exchange bias (i.e., a shift of the hysteresis loop toward positive applied field, where positive means the applied and cooling fields are parallel). ${ }^{20}$ For the case of Co/untwinned single crystalline $\mathrm{FeF}_{2}$, the direction of the pinned magnetization across the FM/AF interface is opposite to the sign of exchange bias. ${ }^{21}$ Furthermore, a net uncompensated magnetization was observed in the AF film bulk when the sample exhibited positive exchange bias. ${ }^{21}$ When the same sample exhibited negative exchange bias, zero net uncompensated magnetization was observed in the AF film bulk. ${ }^{21}$ More recently, application of large positive or negative fields was found to control $\left|H_{E}\right|$ in $\mathrm{Ni}_{1-x} \mathrm{Fe}_{x} \mathrm{~F}_{2} / \mathrm{Co}$ bilayers, in which the $\mathrm{Ni}_{1-x} \mathrm{Fe}_{x} \mathrm{~F}_{2}$ alloy is an AF with a weak anisotropy and significant net un- 
compensated magnetization in the AF bulk. ${ }^{22}$

The importance of spin disorder and uncompensated spins has also been reported for exchange bias systems that are not part of the AF-fluoride family. For example, magneto-optic Kerr effect (MOKE) studies of CoFe/IrMn bilayers suggest that spin disorder at FM/AF interfaces reduces the AF anisotropy. When the reduced anisotropy is taken into account with a modification of the Meiklejohn and Bean model, ${ }^{1,2}$ good quantitative agreement between model and experiment can be achieved. ${ }^{23}$ Measurements of second harmonic generation from $\mathrm{CoO} / \mathrm{Cu} / \mathrm{Fe}$ multilayers have detected uncompensated spins at the $\mathrm{CoO} / \mathrm{Cu}$ interface that are affected by the FM layer even across substantial $\mathrm{Cu}$ layer thickness. ${ }^{24}$

Because of the presumed importance that AF domains have on exchange bias, several experiments have attempted to detect uncompensated magnetization of the domains directly. The relationship between the size of the domain of uncompensated magnetization in the AF and the smallest domain size in the adjacent FM was inferred from scanning MOKE spectroscopy and superconducting quantum interference device (SQUID) magnetometry. The relationship governs the regime of exchange bias [averaging (of FM domains over AF domains) vs nonaveraging] and, hence, the sign and magnitude of exchange bias. ${ }^{25-27}$ Photoemission electron microscopy (PEEM) was used to image antiferromagnetic regions in $\mathrm{LaFeO}_{3}$ with a $\mathrm{Co}$ overlayer, although the sample did not exhibit significant exchange bias. ${ }^{28,29}$ Subsequently, the distribution of sizes of ferromagnetic domains was measured in $\mathrm{Co} / \mathrm{LaFeO}_{3}$ with PEEM in remanence. ${ }^{30}$ Pinned and unpinned moments have been detected near FM/AF interfaces in $\mathrm{Co} /$ untwinned single crystalline $\mathrm{FeF}_{2}$ bilayers using $\mathrm{X}$-ray magnetic circular dichroism, ${ }^{31}$ unpinned moments on both sides of the FM/AF interface using resonant soft $\mathrm{x}$-ray reflectometry (RXR), ${ }^{32}$ and unpinned and pinned moments at the FM/AF interface and in the AF film bulk with neutron reflectometry. ${ }^{21,32}$ RXR measurements of $\mathrm{Fe} / \mathrm{CoO}$ bilayers have also observed different types of uncompensated Co moments that couple parallel or antiparallel to the $\mathrm{Fe}$ moments. ${ }^{33}$ Pinned moments at the FM/AF interface were also reported for $\mathrm{Co} / \mathrm{LaFeO}_{3}$ with neutron reflectometry ${ }^{34}$ and inferred from magnetometry and micromagnetic modeling of $\mathrm{GdFe} / \mathrm{TbFe}$ bilayers. ${ }^{27,35}$

Despite recent successful efforts to quantify the uncompensated magnetization in the AF, measurement of AF domain size has not been reported in exchange bias samples. Here, we report the AF domain sizes for three exchange bias samples obtained from the broadening of AF Bragg reflections measured with neutron diffraction. The first study involved measurement of a Co/untwinned single crystalline $\mathrm{Zn}_{0.30} \mathrm{Fe}_{0.70} \mathrm{~F}_{2}$ bilayer as a function of the magnitude and sign of $H_{E}$. The second study included $\mathrm{Co}$ /untwinned single crystalline $\mathrm{Ni}_{0.55} \mathrm{Fe}_{0.45} \mathrm{~F}_{2}$ and $\mathrm{Co} /$ untwinned single crystalline $\mathrm{FeF}_{2}$ bilayers. Interestingly, while $H_{E}$ is different for all three samples (and as a function of temperature for one sample), and sometimes zero, the AF domain sizes were essentially similar and smaller than the thickness of the AF layers. In fact, the AF domain size was comparable to the lateral dimension of long range order of the atomic lattice.

\section{SAMPLE GROWTH, MAGNETOMETRY, AND X-RAY CHARACTERIZATION}

\section{A. Sample growth}

Growth of Co on epitaxial untwinned single crystalline $\mathrm{FeF}_{2}, \mathrm{Zn}_{1-x} \mathrm{Fe}_{x} \mathrm{~F}_{2}$ (where $x=0.7$ ), or $\mathrm{Ni}_{1-y} \mathrm{Fe}_{y} \mathrm{~F}_{2}$ (where $y$ $=0.45$ ) samples has been described elsewhere. ${ }^{21,36}$ Briefly, samples were prepared by deposition onto the polished surface of large (1-4 $\left.\mathrm{cm}^{2}\right)$ (110) $\mathrm{MgF}_{2}$ single crystals. For the (110) $\mathrm{FeF}_{2}$ film, an electron gun was used to deposit a $\sim 400$ - $\AA$-thick single crystal film. For the (110) $\mathrm{FeF}_{2}$-alloy films, $\mathrm{FeF}_{2}$ and $\mathrm{ZnF}_{2}$ (or $\mathrm{NiF}_{2}$ ) were codeposited using molecular electron beam epitaxy to grow $\sim 600$ - $\AA$-thick single crystal films (or $450 \AA \mathrm{Ni}_{0.55} \mathrm{Fe}_{0.45} \mathrm{~F}_{2}$ ). ${ }^{16}$ The compositions of the alloy films were obtained from measurements of the (001) lattice parameters as described previously. ${ }^{36}$ After growing the alloy film, a $\sim 1$-nm-thick layer of $\mathrm{FeF}_{2}$ was deposited to maintain the same chemical composition locally at the FM/AF interface. Next, polycrystalline films of Co were deposited, followed by Al to prevent oxidation of the Co surface. The deposition temperatures were $300{ }^{\circ} \mathrm{C}$ for the $\mathrm{AF}$ films and $125-150{ }^{\circ} \mathrm{C}$ for the $\mathrm{Co}$ and $\mathrm{Al}$ layers at room temperature.

\section{B. Magnetometry}

To establish exchange bias, the samples were saturated in an external field of $H$ (typically $6 \mathrm{kOe}$ ) applied along [001] $X \mathrm{~F}_{2}$ (where $\left.X=\mathrm{Zn}_{x} \mathrm{Ni}_{y} \mathrm{Fe}_{1-x-y}\right)$ at room temperature $(T$ $=300 \mathrm{~K})$, and then cooled to low temperatures $(\sim 6 \mathrm{~K})$ in a field $H_{\mathrm{FC}}$ (depending upon the experiment, $H_{\mathrm{FC}}$ varied between 0.5 and $5.5 \mathrm{kOe}$ ). Hysteresis loops were measured with a SQUID magnetometer from which $H_{E}$ was obtained. For $T=6 \mathrm{~K}$ and $H_{\mathrm{FC}}=5.5 \mathrm{kOe}, H_{E}= \pm 1750$ and $+480 \mathrm{Oe}$ for the $\mathrm{Co} / \mathrm{FeF}_{2}$ and $\mathrm{Co} / \mathrm{Ni}_{0.55} \mathrm{Fe}_{0.45} \mathrm{~F}_{2}$ samples, respectively. The temperature and cooling field dependence of $H_{E}$ for $\mathrm{Co} / \mathrm{Zn}_{0.30} \mathrm{Fe}_{0.70} \mathrm{~F}_{2}$ is more complicated than that for the other samples. When cooled in fields $H_{\mathrm{FC}}=6 \mathrm{kOe}$, the exchange bias was negative at low temperature, equal to zero for $20 \mathrm{~K}$, and then became positive above $20 \mathrm{~K}$ (red squares, Fig. 1) similar to what was reported previously. ${ }^{16}$ Thus, by choosing temperature, we were able to investigate the relationship between the AF domain state and $H_{E}$ for the same sample.

\section{X-ray reflectometry and glancing incidence x-ray diffraction}

For each sample, individual layer thickness and interface roughness were determined using $\mathrm{x}$-ray reflectometry. Reflectometry involves measurement of the radiation ( $\mathrm{x}$ rays or neutrons) reflected from a sample (Fig. 2) as a function of wave vector transfer $Q$ (i.e., the difference between the outgoing and incoming wave vectors) and the intensity of the incident beam. The most intensely reflected beam corresponds to the specular reflectivity where the angle of reflection from the surface $\alpha_{f}$ and the angle of incidence $\alpha_{i}$ are equal [Fig. 2(a)]. In addition, diffusely scattered radiation (e.g., as produced by rough surfaces) may also be observed [Fig. 2(b)]. ${ }^{37-39}$ 


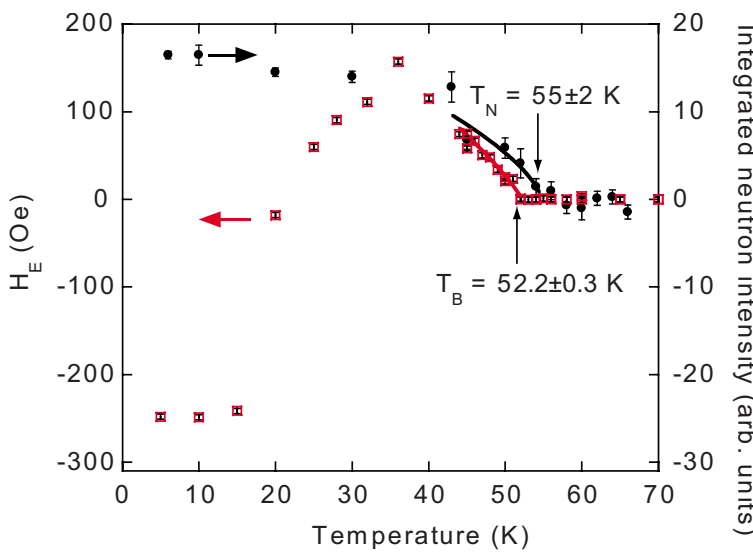

FIG. 1. (Color online) Exchange bias $H_{E}(\square)$ as a function of temperature for the $\mathrm{Co} / \mathrm{Zn}_{0.30} \mathrm{Fe}_{0.70} \mathrm{~F}_{2}$ sample. The temperature dependence of the integrated intensity of the (100) Bragg reflection are shown $($ ). The solid curves are fits to a model discussed in the text.

Measurements of the scattering by the samples over a large range of wave vector transfer parallel, $Q_{x}$, and perpendicular, $Q_{z}$, to the sample's surface (Fig. 3, upper panels) were made using $\mathrm{Cu} K_{\alpha}$ x rays. To obtain the specular reflectivity (corresponding to the intensity at $Q_{x}=0$ in Fig. 3), we subtracted an estimate for the contribution of diffuse scattering at $Q_{x}=0$ using measurements where $Q_{x} \neq 0$ (Fig. 3, lower panels). The specular reflectivities (symbols, Fig. 3) were fitted to models (Fig. 3, inset) using the dynamical formalism of Parratt. ${ }^{40-42}$ The best-fitting models, yielding the calculated reflectivities (red curves, Fig. 3), were ones that minimized the $\chi^{2}$ metric. $^{43,44}$

(a)

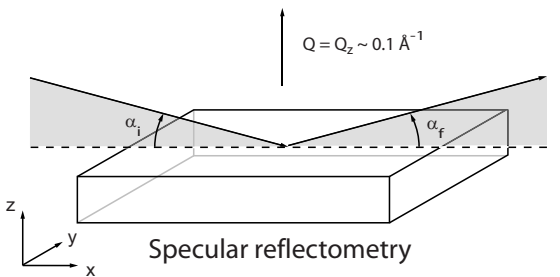

(b)

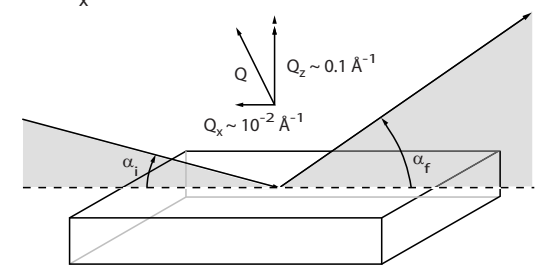

Off-specular reflectometry

(c)

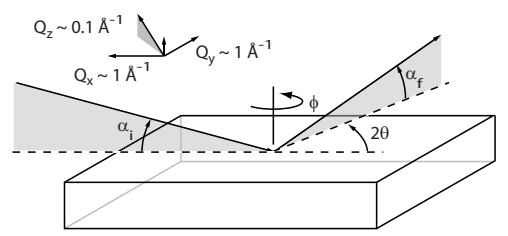

Glancing incidence diffraction

FIG. 2. Schematics of (a) specular reflectometry, (b) offspecular reflectometry, and (c) glancing incidence (x-ray) diffraction. These geometries were used for the $\mathrm{x}$-ray diffraction experiments. Insets: Diagrams showing typical values for the wave vector transfer for the experiments.
Glancing incidence x-ray diffraction (GIXD) (Ref. 45) [Fig. 2(c)] was used to characterize the atomic structure of the $\mathrm{FeF}_{2}$ film in the $\mathrm{Co} / \mathrm{FeF}_{2}$ bilayer sample. Briefly, this technique involves illuminating the sample at an angle of incidence near the critical angle of the sample $\left(\alpha_{i}=\alpha_{c}\right.$ $=\lambda \sqrt{\rho / \pi}$, where $\lambda=1.54 \AA$ is the wavelength of the $\mathrm{x}$ rays and $\rho$ is electron density times the Bohr radius). A position sensitive $\mathrm{X}$-ray detector was moved about the sample's surface normal through an angle $2 \theta$ corresponding to twice the Bragg angle for the in-plane Bragg reflections of interest [Fig. 2(c)]. The sample was rotated $\phi$ about its surface normal.

We measured the intensity of the scattered radiation as a function of $\alpha_{f}$ and $\phi$ for the $\mathrm{Co} / \mathrm{FeF}_{2}$ sample [Fig. 4(a)]. ${ }^{46}$ Integrating the intensity within $0.2^{\circ}$ of $\phi_{(\overline{1} 10)}$ yielded the intensity profile vs $\alpha_{f}$ [Fig. 4(b)] that is characteristic of scattering from a crystalline surface or interface. ${ }^{45}$ On the other hand, integrating the intensity in the range of $\alpha_{f} / \alpha_{c}$ from 0.5 to 1.5 yielded the intensity profile vs $\phi[$ Fig. $4(\mathrm{c})]$, which represents the scattering from the portion of the $\mathrm{FeF}_{2}$ film near the FM/AF interface. ${ }^{45}$ The two peaks of intensity separated by $180^{\circ}$ correspond to the $(1 \overline{10})$ and $(\overline{1} 10) \mathrm{FeF}_{2}$ Bragg reflections, thus confirming that the $\mathrm{FeF}_{2}$ film is single crystalline. One measure of single crystalline quality is the inplane mosaic of the $\mathrm{FeF}_{2}$ film given by the width $\delta \phi \sim 0.4^{\circ}$ (rms) of the (1 $\overline{1} 0) \mathrm{FeF}_{2}$ Bragg reflection. For comparison, the in-plane mosaic of the surface of an as-received polished single crystal $\mathrm{MgF}_{2}$ substrate was $0.26^{\circ}$ (rms). The out-ofplane mosaic of the same substrate obtained from the (110) Bragg reflection was $0.06^{\circ}$ (rms).

A second measure of crystalline quality is the width of the Bragg reflection along the longitudinal direction (the direction corresponding to a change of $|Q|$ but not its orientation). This width was affected by the resolution of the diffractometer and sources of broadening from portions of the sample that coherently scattered the radiation. Even for a single crystal, defects, such as dislocations and low angle grain boundaries, can limit the size over which the atomic structure exhibits long range order. ${ }^{47}$ We call this size the crystal domain size $\Delta_{\text {xtal }}$.

To obtain $\Delta_{\text {xtal }}$, we measured the widths in $Q$ of the (220), (400), and (440) Bragg reflections from a perfect GaAs crystal using longitudinal scans under conditions of GIXD. These widths [full width at half maximum (FWHM)] are shown by the open symbols in Fig. 4(d). We used the average value as a measure of the resolution $\left(\sigma_{\text {ins }}=0.007 \AA^{-1} \mathrm{rms}\right)$ of our $\mathrm{X}$-ray diffractometer. Next, we recorded the longitudinal scans for the (110), (220), and (330) Bragg reflections from the $\mathrm{FeF}_{2}$ film. After accounting for the broadening intrinsic to the instrument, ${ }^{48}$ we obtained the widths $\Gamma$ (FWHM) for the $\mathrm{FeF}_{2}$ Bragg reflections [closed symbols in Fig. 4(d)]. $\Delta_{\mathrm{xtal}}$ and the microstrain broadening $\varepsilon$ are related to $\Gamma$ and $Q$ via the relation ${ }^{49}$

$$
\Gamma=2 \pi / \Delta_{\mathrm{xtal}}+\sqrt{\varepsilon_{(110)}^{2}} Q
$$

A fit of Eq. (3) minimizing the $\chi^{2}$ metric [line in Fig. 4(d) $]^{50}$ yields $\Delta_{\text {xtal }}=310 \pm 30 \AA$, and $\sqrt{\varepsilon_{(110)}^{2}}=0.55 \% \pm 0.05 \%$. The 
(a) $\mathrm{Co} / \mathrm{Ni}_{0.55} \mathrm{Fe}_{0.45} \mathrm{~F}_{2}$

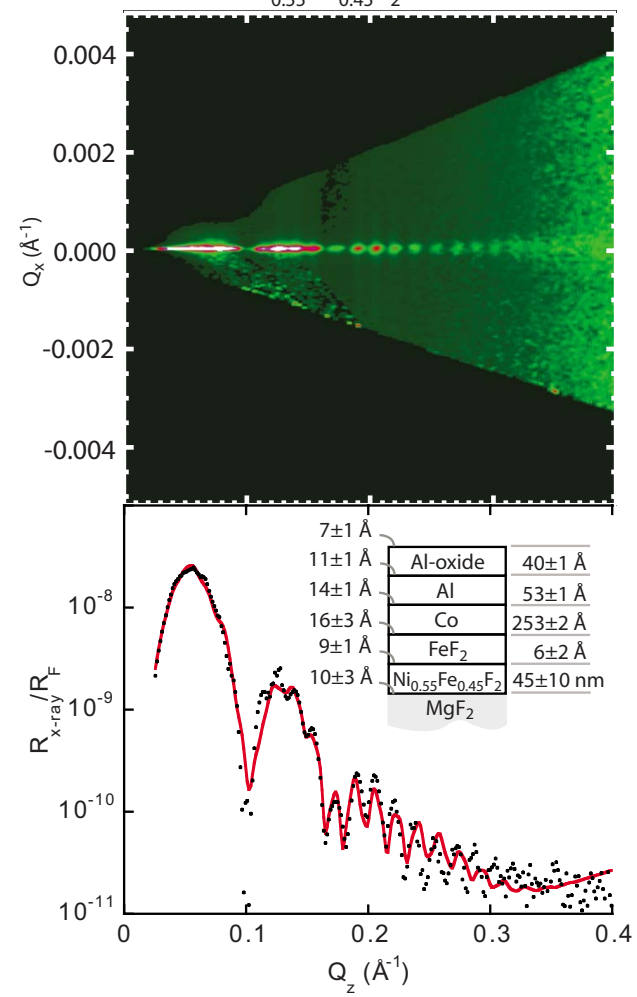

(b) $\mathrm{Co} / \mathrm{Zn}_{0.3} \mathrm{Fe}_{0.7} \mathrm{~F}_{2}$

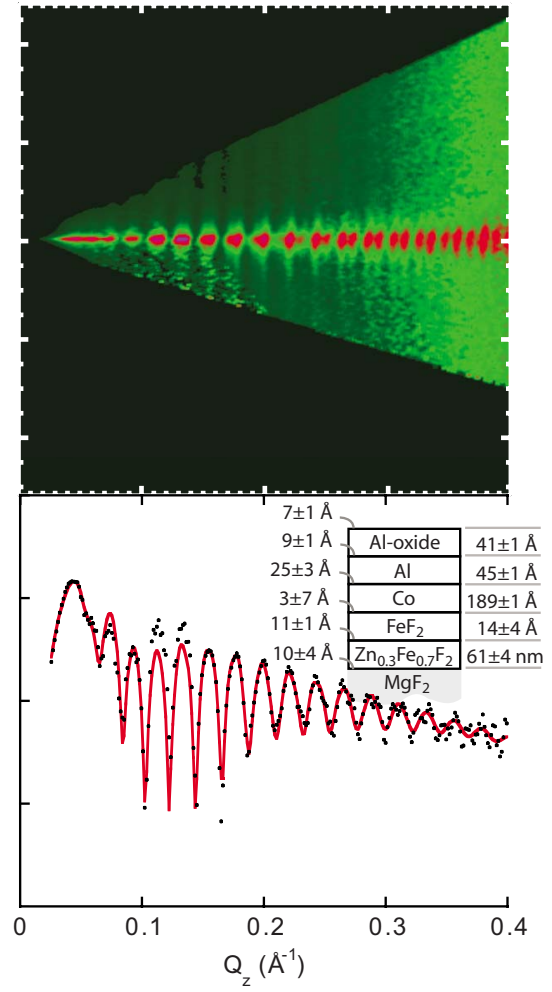

(c) $\mathrm{Co} / \mathrm{FeF}_{2}$

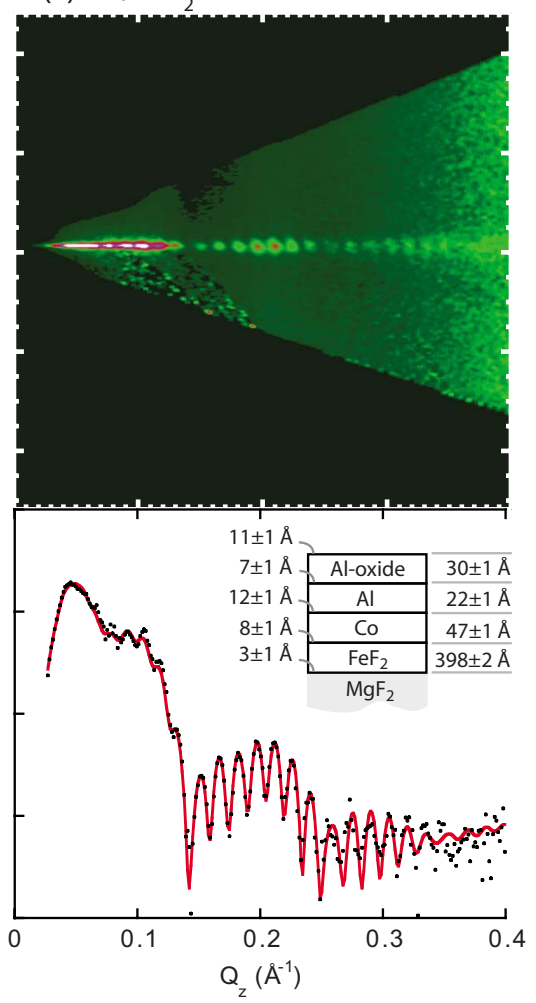

FIG. 3. (Color online) (Upper panels) Off-specular and specular x-ray intensity maps and (lower panels) reflectivities normalized to the Fresnel reflectivity $\left(R_{F}=16 \pi^{2} / Q_{z}^{4}\right)$ for the three samples (a) $\mathrm{Co} / \mathrm{Ni}_{0.55} \mathrm{Fe}_{0.45} \mathrm{~F}_{2}$, (b) $\mathrm{Co} / \mathrm{Zn}_{0.30} \mathrm{Fe}_{0.70} \mathrm{~F}_{2}$, and (c) Co/ FeF . The solid curves are the best-fitting reflectivities calculated using the chemical model shown in the inset.

x-ray result for $\Delta_{\text {xtal }}$ is a measure of the lateral (i.e., across the sample's surface) crystal domain size.

Previously, the dislocation density at the $\mathrm{Co} / \mathrm{FeF}_{2}$ interface (dislocations help relieve misfit strain between the $\mathrm{AF}$ film and substrate $)^{51}$ was measured with transmission electron microscopy and found to be about half of what would be expected to fully relieve the mismatch between the lattice parameters of $\mathrm{FeF}_{2}$ and $\mathrm{MgF}_{2} \cdot{ }^{32,52,53}$ Here, we have shown that microstrain is indeed present in the $\mathrm{FeF}_{2}$ film. Since $\mathrm{FeF}_{2}$ is a piezomagnetic material, ${ }^{54}$ microstrain may produce uncompensated magnetization even in pure films. ${ }^{55,56}$

\section{NEUTRON SCATTERING EXPERIMENT}

The neutron-scattering experiments used the Asterix spectrometer at the Los Alamos Neutron Science Center-a short-pulsed spallation neutron source. The Asterix spectrometer is a reflectometer and diffractometer that provides polarized or unpolarized neutron beams. We used the unpolarized neutron beam. The spectrometer views a partially coupled cold neutron moderator ${ }^{57}$ through a ${ }^{58} \mathrm{Ni}$ guide.$^{58}$ Neutrons scattered by a sample were detected, and the scattering angle in the horizontal plane $2 \theta$ was measured using one $(20 \mathrm{~cm}$ long $)$ and two-dimensional $\left(20 \times 20 \mathrm{~cm}^{2}\right)$ position sensitive detectors located $0.62 \mathrm{~m}$ from the sample. Neutron wavelength $\lambda$ (ranging from 1 to $10+\AA$ ) was measured using the time-of-flight technique. ${ }^{42}$

The orientation of the unit cell of the AF with respect to the incident neutron beam is shown in Figs. 5(a) and 5(b) (the $\mathrm{MgF}_{2}$ substrate is omitted for clarity). The Fe spins are represented by arrows that are color coded according to whether the spin sublattice points up (blue) or down (red) [Fig. 5(a)]. The color-coding scheme is carried forward in Figs. 5(b) and 5(c) without explicitly showing the Fe spins. In Fig. 5(b), the incident neutron beam wave vector $k_{i}$ is directed toward the reader and strikes the sample's (110) surface. The wave vector of the diffracted radiation then travels to the reader's right. If the reader's perspective is moved to look down upon the top surface of the threedimensional rendering in Fig. 5(b), the plan-view schematic shown in Fig. 5(c) is obtained.

The orientation of a sample was chosen such that the neutron beam struck the sample at near normal incidence (i.e., the complement of the angle of incidence was $\omega \sim-1^{\circ}$ ). For this orientation, the wave vector transfer $Q(=2 \pi / d$, where $d$ is $d$ spacing) for the (100) Bragg reflection was $\sim 46^{\circ}$ from the sample's surface normal, requiring the detector to be at $2 \theta \sim 88^{\circ}$. The single crystal structures of our samples select a single wavelength for each $[h 00]$ Bragg reflection. Since our spectrometer provides neutrons with a broad range of wavelength, Bragg's law could always be satisfied. The position $2 \theta \sim 88^{\circ}$ of the detector is particularly favorable since the detector "viewed" the sample along its edge. Thus, the divergence of the scattered (diffracted) neutron beam was limited by the mosaic spread of the sample $\delta \phi$ (Fig. 5), ${ }^{59}$ rather than being worsened by the projection of a large sample footprint onto the detector. The ability to orient the sample at near normal incidence and to position the detector at $90^{\circ}$ meant 

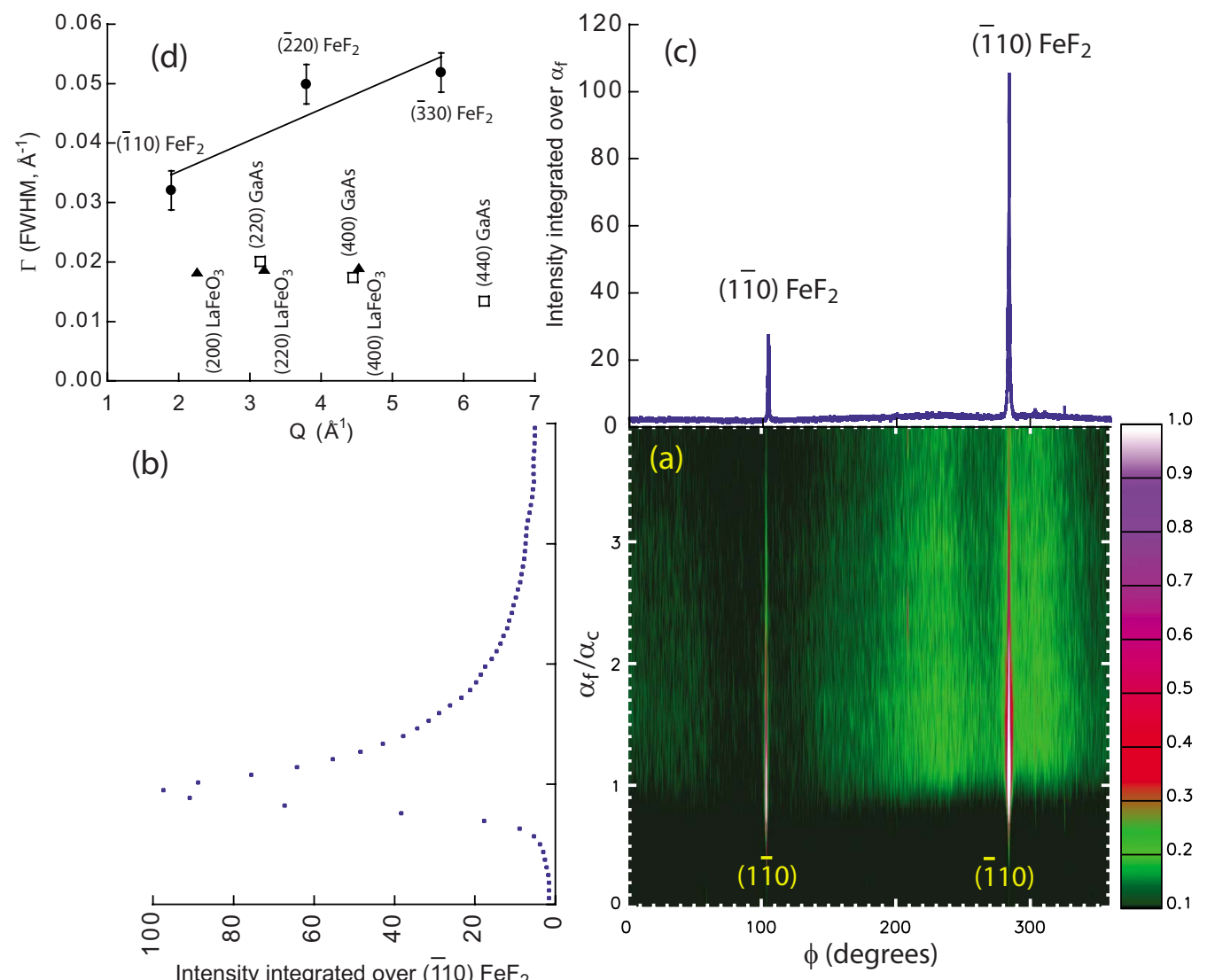

FIG. 4. (Color online) (a) X-ray intensity map measured under conditions of glancing incidence diffraction for the (110)-type Bragg reflection from the $\mathrm{FeF}_{2}$ film. (b) X-ray intensity profile of the (110) Bragg reflection vs $\alpha_{f}$. (c) X-ray intensity profile integrated over a region corresponding to $\alpha_{f} / \alpha_{c}=1.0 \pm 0.5$ vs $\phi$. (d) Solid symbols: Width (FWHM) of the (hh0) Bragg reflections for FeF $F_{2}$ film (and for select Bragg reflections from the $\mathrm{LaFeO}_{3}$ film and a $\mathrm{GaAs}$ perfect crystal, open symbols). The line is the best fit of Eq. (1) to the FeF 2 data.

that a neutron beam with the largest possible cross section (equal to the surface area of the sample) ${ }^{60}$ and divergence could be used to maximize the intensity of the (100) Bragg reflection without compromising resolution (needed to measure $d$ ). These advantages enabled detection of the AF Bragg reflections from thin single crystal films with masses of $<10 \mu \mathrm{g}-$ much less than used for most neutron diffraction experiments.

In addition to offering the opportunity to optimize the diffraction geometry to detect scattering from our samples, the time-of-flight technique offers a critically important advantage over another approach to measure $\lambda$-one that uses crystal monochromators. Crystal monochromators select a fundamental wavelength $\lambda$ and its harmonics (i.e., $\lambda / 2, \lambda / 3$, ...). Neutron filters are available for crystal monochromators that attenuate the harmonics of the fundamental radiation by factors of 100 or so. ${ }^{61}$ This attenuation factor is inadequate for our studies. Since the substrate-to-film mass ratio is typically on the order of $10^{5}$, the nuclear scattering from a higher order Bragg reflection using a harmonic of the fundamental wavelength would be comparable to the magnetic scattering from our film (the two nearly coincide because the lattice parameters are reasonably similar-a requirement for epitaxial film growth). For example, the integrated intensity of the magnetic reflection from a $\mathrm{NiF}_{2}$ film was measured as a function of temperature, but a residual background was ob- (a)

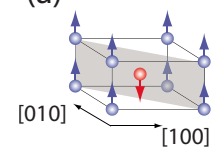

(b)

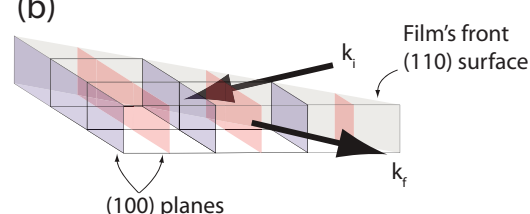

(c)

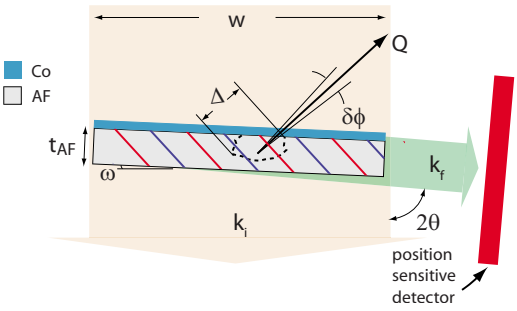

FIG. 5. (Color online) (a) Unit cell of the AF color coded to show the two spin sublattices. (b) Three-dimensional perspective of the incident neutron wave vector $k_{i}$ illuminating the sample's surface [the (110) plane] from behind the figure and the diffracted wave vector $k_{f}$ exiting the figure at right. (c) Schematic of the neutron-scattering experiment as viewed from above (b). A neutron beam $(\tan )$ with width $w$ illuminates the sample. A position sensitive detector measures the neutron intensity as a function of $2 \theta$ and wavelength. An AF domain with dimension $\Delta$ parallel to the wave vector transfer $Q$ is shown. The sample's surface normal is rotated from the incident neutron beam by an angle $\omega$. 


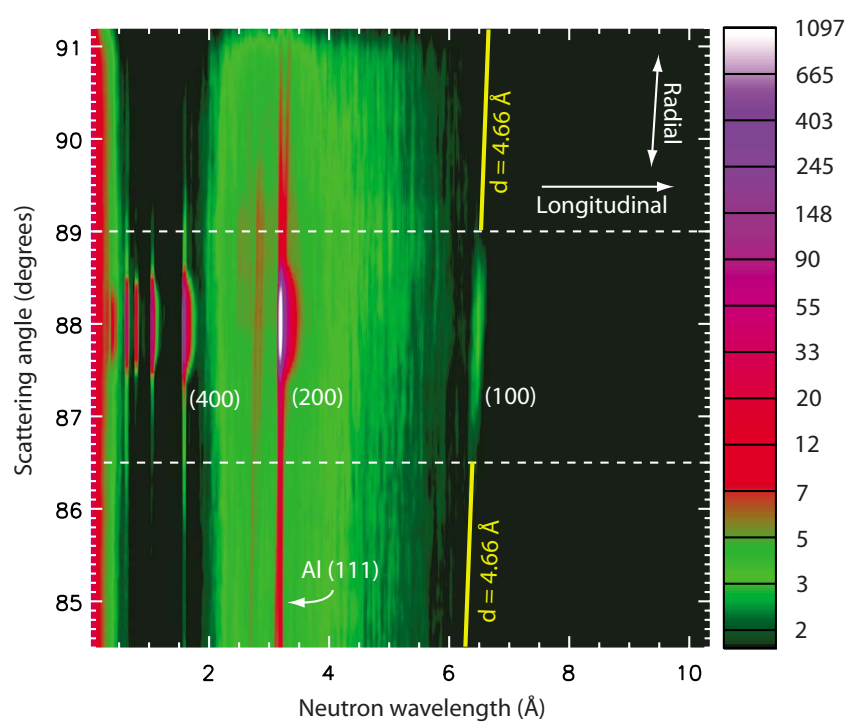

FIG. 6. (Color online) Neutron intensity image (neutrons counted in a bin with dimensions $0.005 \AA$ by $0.066^{\circ}$, scale at right) vs $2 \theta$ and $\lambda$. ( $h 00)$-type Bragg reflections are labeled. The intensity within the dashed box was integrated along contours of constant $d$ spacing to form the diffraction pattern in Fig. 7. A contour of constant $d$ spacing $(d=4.66 \AA)$ is shown by the yellow line.

tained as a result of harmonic contamination from the substrate. ${ }^{62}$ This difficulty does not occur for time-of-flight experiments.

The neutron intensity measured for the $\mathrm{Co} / \mathrm{Zn}_{0.30} \mathrm{Fe}_{0.70} \mathrm{~F}_{2}$ sample at $T=6 \mathrm{~K}$ is shown in Fig. 6 versus time of flight and scattering angle. The sharp peaks for $\lambda<4 \AA$ are Bragg reflections primarily from the single crystal $\mathrm{MgF}_{2}$ substrate (the thin film contributes too, but at a factor roughly equal to the ratio of film-to-substrate thickness of $\sim 7 \times 10^{-5}$ ). The weak peak indexed as (100) is a Bragg reflection from the antiferromagnetic order of the $\mathrm{Zn}_{0.30} \mathrm{Fe}_{0.70} \mathrm{~F}_{2}$ film. The long streak of intensity at $d=2.33 \AA$ (from small to large $2 \theta$ ) corresponds to a contour of constant $d$ spacing. This streak of intensity is a portion of the (111) Debye-Scherrer ring from the $\mathrm{Al}$ cryostat shrouds. Integrating the neutron intensity along contours of constant $d$ spacing for $2 \theta$ confined within the dashed lines in Fig. 6 yields the diffraction pattern for (h00) Bragg reflections [Fig. 7(a)]. This diffraction pattern was obtained in $\sim 9 \mathrm{~h}$.

The (rms) width $\Sigma$ (in $d$ spacing) of a Bragg reflection was obtained from fits of a Gaussian function [e.g., a solid curve Fig. $7($ b) $]$ to the intensity profile. $\Sigma$ contains contributions from uncertainty in measurement of $d$ spacing $\delta d$ (i.e., the resolution of the neutron spectrometer) and the sample (e.g., domain size broadening). To account for uncertainties in measurements of neutron wavelength $\delta \lambda$ and neutron trajectory $\delta \theta$ contributing to $\delta d$ we used the relation ${ }^{63,64}$

$$
\begin{aligned}
\left(\frac{\delta d}{d}\right)^{2} & =\left[(\delta \phi)^{2} \cot ^{2} \theta+\left(\frac{\delta \lambda}{\lambda}\right)^{2}\right] \\
& =\left[(\delta \phi)^{2} \cot ^{2} \theta+\left(\frac{\sigma_{m}}{t}\right)^{2}\right] .
\end{aligned}
$$

The geometrical contribution (the first term in brackets) is determined by the scattering angle where the (h00) reflections are observed, $2 \theta \approx 88^{\circ}$, and the (rms) mosaic spread of the AF thin film $\delta \phi \approx 0.4^{\circ}\left(\delta \phi \approx 0.06^{\circ}\right.$ for the $\mathrm{MgF}_{2}$ substrate). This contribution is $\sim 5 \times 10^{-5}$.

Since the neutron time of flight $t$ is proportional to neutron wavelength $\lambda, \delta \lambda$ is related to the (rms) time constant $\sigma_{m}$ of the coupled $l-\mathrm{H}_{2}$ moderator that produced the cold neutron beam. ${ }^{57,65,66}$ To obtain the time-of-flight contribution [the second term in brackets in Eq. (2)], we measured the intensity profile of the (002) graphite Bragg reflection (Fig. $8)$. Graphite was chosen since the (002) reflection could be measured with a wavelength $(\lambda \sim 6.3 \AA)$ comparable to that $(\lambda \sim 6.5 \AA)$ used for the (100) reflections from our samples. ${ }^{67}$ The measurement was made in a separate experiment that first involved reflecting the neutron beam at glancing angle from a Si wafer and then measuring the (002) graphite reflection in back-reflection geometry. ${ }^{68}$ To extract

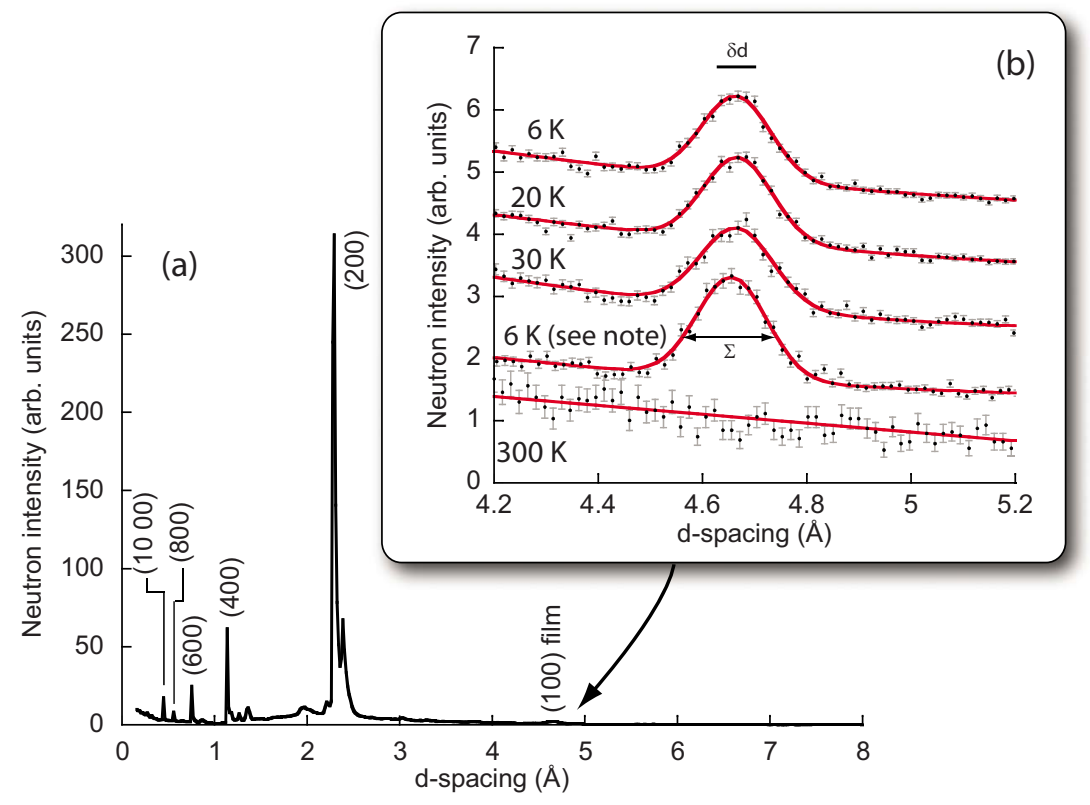

FIG. 7. (Color online) (a) (h00)-type diffraction pattern at 6 K. (b) The (100) $\mathrm{Zn}_{0.30} \mathrm{Fe}_{0.70} \mathrm{~F}_{2}$ AF Bragg reflection profile for $T$ =6, 20, 30, and $300 \mathrm{~K}$. The solid curves are Gaussian functions fitted to the scans. Note that the bottom $6 \mathrm{~K}$ measurement was taken with $H_{\mathrm{FC}}$ applied perpendicular to the easy axis of the AF. 


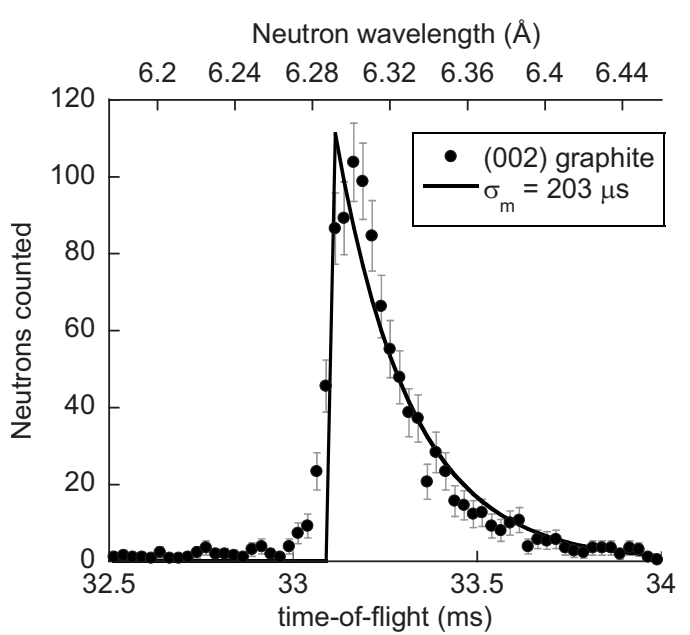

FIG. 8. The (002) graphite Bragg reflection measured as a function of neutron time of flight. The solid curve is the intensity profile of a function proportional to $e^{-\left|t-t_{0}\right| / \sigma_{m}}$ (for $t>t_{0}$ ) where $t$ is the time of flight and $\sigma_{m}$ is the moderator time constant (emission time of the moderator after $t_{0}$ ).

$\sigma_{m}$, we fitted the (002) graphite profile to the function $e^{\left|t-t_{0}\right| / \sigma_{m}}$ for $t>t_{0}$ (or 0 for $t<t_{0}$ ) (solid curve, Fig. 8) and obtained $\sigma_{m}=203 \mu \mathrm{s}$. Thus, the wavelength contribution to the uncertainty $(\delta d / d)^{2}$ was $\sim 6 \times 10^{-5}$ for $\lambda \sim 6.3 \AA$. Together with the geometrical contribution, $\delta d / d=0.01$. For the range of $d$ spacing shown in Fig. $7, \delta d=0.047 \AA$ and represents the (rms) instrumental contribution to the broadening of the (100) AF Bragg reflection.

\section{RESULTS}

\section{A. Characteristic dimensions of the antiferromagnetic domain state $\mathrm{Zn}_{0.30} \mathrm{Fe}_{\mathbf{0 . 7 0}} \mathrm{F}_{2}$ for different $H_{E}$}

Appearance of the reflection below the Néel temperature of $\mathrm{Zn}_{0.30} \mathrm{Fe}_{0.70} \mathrm{~F}_{2}\left(T_{N}=55 \mathrm{~K}\right.$; discussed later $)$ and its absence at $300 \mathrm{~K}$ [Fig. 7(b)] suggests the reflection is a consequence of ordering of the AF sublattices (depicted by red and blue lines in Fig. 5). ${ }^{69}$ Broadening of the reflection in excess of the instrumental contribution $\sqrt{\Sigma^{2}-(\delta d)^{2}}$ is a measure of the volume-averaged size $\Sigma_{100}$ of coherently scattering domains along a direction of $\sim 45^{\circ}$ from the sample's surface normal. ${ }^{70}$ Since the (100) Bragg reflection is exclusively due to magnetic order of the spin lattice, the domain size obtained from its width is representative of the AF domains. ${ }^{71}$
Re-expressing Eq. (1) in terms of peak widths measured in $d$ spacing yields ${ }^{72}$

$$
\sqrt{8 \ln 2} \Sigma_{(100)}=d_{(100)}^{2} / \Delta_{(100)}+d_{(100)} \sqrt{\varepsilon_{(100)}^{2}},
$$

where $d_{(100)}=4.67 \AA$ is the $d$ spacing for the (100) Bragg reflection, $\Delta_{(100)}$ is a volume-averaged domain size (parallel to $Q$ ), $\sqrt{\varepsilon_{(100)}^{2}}$ is the volume-averaged microstrain (parallel to $Q)$, and the factor of $\sqrt{8 \ln 2}$ accounts for the fact that $\Sigma$ is an rms quantity and $\Gamma$ in Eq. (1) is a FWHM. ${ }^{49}$ Owing to the similar lattice parameters of the film and $\mathrm{MgF}_{2}$, the nuclear Bragg reflections from the film and substrate coincide. Therefore, we were unable to quantify $\sqrt{\varepsilon_{(100)}^{2}}$. This shortcoming, however, is not problematical since for large $d$ spacings, domain size broadening dominates microstrain broadening [see Eq. (1) in the limit where $Q \rightarrow 0$ ]. If we assume the strain broadening of the (100) AF Bragg reflection is equivalent to the strain broadening obtained with GIXD for the (110) reflection from the $\mathrm{Co} / \mathrm{FeF}_{2}$ sample, ${ }^{73}$ then $\sqrt{\varepsilon_{(100)}^{2}} \approx \sqrt{\varepsilon_{(110)}^{2}},{ }^{74}$ and the AF domain size is

$$
\begin{aligned}
\Delta_{(100)} & =\frac{d_{(100)}^{2}}{\sqrt{8 \ln 2} \Sigma_{(100)}-d_{(100)} \sqrt{\varepsilon_{(100)}^{2}}} \\
& \approx \frac{d_{(100)}^{2}}{\sqrt{8 \ln 2} \sqrt{\Sigma^{2}-(\delta d)^{2}}-d_{(100)} \sqrt{\varepsilon_{(110)}^{2}}} .
\end{aligned}
$$

The AF domain size along [100], $\Delta_{(100)}$, is summarized in Table I for the $\mathrm{Co} / \mathrm{Zn}_{0.30} \mathrm{Fe}_{0.70} \mathrm{~F}_{2}$ sample as a function of temperature and $H_{E}$. Note that the last entry in Table I (corresponding to $H_{E}=0$ Oe at $T=6 \mathrm{~K}$ ) was obtained by cooling the sample with the field applied perpendicular to the easy axis of the AF sublattice.

\section{B. Characteristic dimensions of the antiferromagnetic domain state for different alloy compositions}

The previous experiment compared the AF domain size for the same sample as a function of temperature and exchange bias. Next, we present data (Fig. 9) for the (100) AF Bragg reflections of similarly cooled samples $\left(H_{\mathrm{FC}}\right.$ $=5.5 \mathrm{kOe} \|[001]$, and $T=6 \mathrm{~K})$ with different alloy compositions $\left(\mathrm{Co} / \mathrm{Ni}_{0.55} \mathrm{Fe}_{0.45} \mathrm{~F}_{2}, \mathrm{Co} / \mathrm{Zn}_{0.30} \mathrm{Fe}_{0.70} \mathrm{~F}_{2}\right.$, and $\left.\mathrm{Co} / \mathrm{FeF}_{2}\right)$. These data were collected using a one-dimensional position sensitive detector with $1 \mathrm{~mm}$ wide pixels and time-of-flight bins $25 \mu$ s wide, rather than the two-dimensional detector

TABLE I. Temperatures $T$ and exchange bias $H_{E}$ corresponding to the neutron-scattering measurements. The AF domain size $\Delta$ parallel to [100] $\mathrm{Zn}_{0.30} \mathrm{Fe}_{0.70} \mathrm{~F}_{2}$ was obtained from the width $\Sigma$ (rms) of the (100) AF Bragg reflection (Fig. 7). For these measurements $2 \theta=80^{\circ}$ and $\delta d=0.052 \AA$.

\begin{tabular}{cccccc}
\hline \hline $\begin{array}{c}T \\
(\mathrm{~K})\end{array}$ & $\begin{array}{c}H_{E} \\
(\mathrm{Oe})\end{array}$ & $\begin{array}{c}H_{C} \\
(\mathrm{Oe})\end{array}$ & $\begin{array}{c}\Delta_{100} \\
(\AA)\end{array}$ & $\begin{array}{c}\sum_{\AA} \\
(\AA)\end{array}$ & Cooling field orientation \\
\hline 6 & -234 & 45 & $322 \pm 51$ & $0.066 \pm 0.004$ & $H_{\mathrm{FC}} \|[001]$ \\
20 & 0 & 232 & $322 \pm 51$ & $0.066 \pm 0.004$ & $H_{\mathrm{FC}} \|[001]$ \\
30 & +101 & 134 & $265 \pm 42$ & $0.070 \pm 0.005$ & $H_{\mathrm{FC}} \|[001]$ \\
6 & 0 & & $305 \pm 46$ & $0.067 \pm 0.004$ & $H_{\mathrm{FC}} \perp[001]$ \\
\hline \hline
\end{tabular}




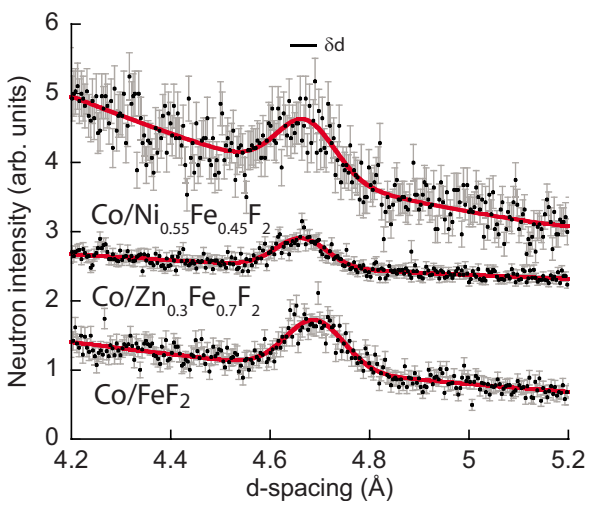

FIG. 9. (Color online) The (100) AF Bragg reflection profile for AF films with different alloy compositions. Profiles are displaced for the sake of clarity, and only a portion of the data for $d$ spacing ranging from 0.2 to $8 \AA$ are shown. Approximately $2 \mathrm{~h}$ were required for each measurement.

with $2 \times 2 \mathrm{~mm}^{2}$ wide pixels and time-of-flight bins $100 \mu \mathrm{s}$ wide that was used to collect the data in Fig. 7. $\Delta_{(100)}$ is given in Table II for samples using different AF alloy compositions measured with the same experimental setup.

\section{Temperature dependence of antiferromagnetic ordering in $\mathbf{Z n}_{0.30} \mathrm{Fe}_{\mathbf{0 . 7 0}} \mathrm{F}_{2}$}

The integrated intensity of the (100) $\mathrm{Zn}_{0.30} \mathrm{Fe}_{0.70} \mathrm{~F}_{2}$ Bragg reflection was also measured as a function of temperature. The $\mathrm{Co} / \mathrm{Zn}_{0.30} \mathrm{Fe}_{0.70} \mathrm{~F}_{2}$ sample was cooled to $6 \mathrm{~K}$ in a field of $H_{\mathrm{FC}}=5.5 \mathrm{kOe}$. The applied field was cycled between $\pm 5.5 \mathrm{kOe}$, and then the diffraction pattern was measured as the sample was warmed through $T_{N}$ in a field of $5.5 \mathrm{kOe}$. To obtain the integrated intensity, a quadratic polynomial function (green curve, Fig. 10) was fitted to the intensity profile shown by the black symbols in Fig. 10. The integrated intensity (corresponding to the area between the blue symbols and green curve, Fig. 10) was obtained by summing the difference between the intensities and the estimate of the background for $\left|d-d_{(100)}\right| \leq 0.17 \AA$. The temperature dependence of the (100) $\mathrm{Zn}_{0.30} \mathrm{Fe}_{0.70} \mathrm{~F}_{2}$ Bragg reflection is shown in Fig. 1 (black circles). The black solid curve in Fig. 1 is the square of the sublattice magnetization given by $M_{\mathrm{AF}}^{2} \propto\left(T_{N}-T\right)^{2 \beta}$ for $43 \mathrm{~K} \leq T \leq T_{N}$, for $\beta=0.33$. $^{75,76}$ The power of " 2 " in the expression for $M_{\mathrm{AF}}$ is required because the integrated intensity of an AF Bragg reflection is proportional to $M_{\mathrm{AF}}^{2}{ }^{77-79} T_{N}$ and

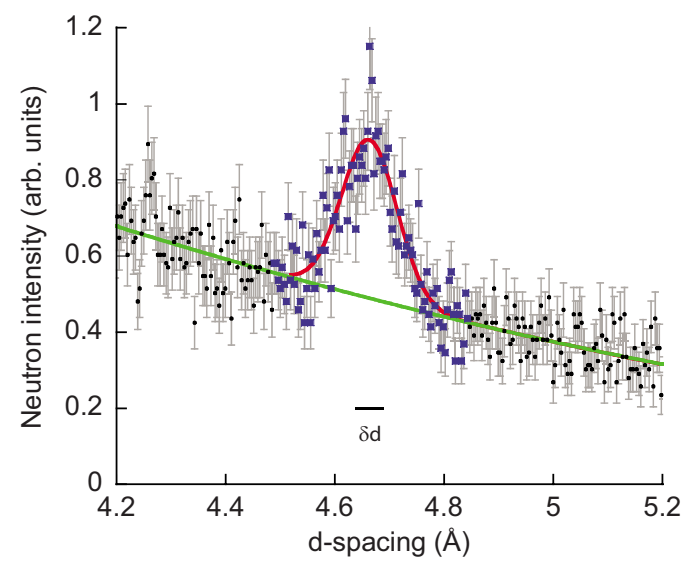

FIG. 10. (Color online) The (100) $\mathrm{Zn}_{0.30} \mathrm{Fe}_{0.70} \mathrm{~F}_{2}$ AF Bragg reflection profile at $6 \mathrm{~K}$ showing the intensities (blue squares) and fitted background (green curve) whose difference is the integrated intensity of the reflection.

a constant of proportionality were optimized to minimize the $\chi^{2}$ metric, ${ }^{43}$ yielding $T_{N}=55 \pm 2 \mathrm{~K}$.

The temperature dependence of $H_{E}$ was determined after cooling in $H_{\mathrm{FC}}=6 \mathrm{kOe}$ from measurements of hysteresis loops. In this case, $H_{E}$ was fitted to the expression $H_{E} \propto(1$ $\left.-T / T_{B}\right)^{\beta^{\prime}}$ for $45 \mathrm{~K} \leq T \leq T_{B}$ with measurements taken every $1 \mathrm{~K}$, where $T_{B}$ is the blocking temperature (temperature above which $H_{E}=0$ ). The best fit (Fig. 1, red solid curve) yielded $\beta^{\prime}=0.8 \pm 0.1$ and $T_{B}=52.2 \pm 0.3 \mathrm{~K}$. The value of $\beta^{\prime}$ agrees with that of pure $\mathrm{FeF}_{2},{ }^{80}$ which is consistent with the surface ordering exponent (0.80) for the Ising model for samples with large grains. ${ }^{80}$

\section{DISCUSSION}

\section{A. Consistently small AF domains}

One remarkable aspect of our results is that within the uncertainty of our measurements, the AF domain size of the bulk film is mostly unchanged regardless of exchange bias in the same sample. Certainly, $H_{E}$ is not inversely proportional to the AF domain size. From Table I we see for the same sample exhibiting different $H_{E}$, the characteristic dimensions of antiferromagnetic domains are on the order of $300 \AA$ along a direction at $45^{\circ}$ to the sample surface. Since this dimension is smaller than the 400-610 $\AA$ thicknesses of the AF films, the AF domains may be laterally constrained. We note that measurement of the lateral crystal domain size was

TABLE II. $H_{E}$ (and interface coupling constant $J_{\text {int }}$ ) at $T=6 \mathrm{~K}$ for $H_{\mathrm{FC}}=5.5 \mathrm{kOe} \|[001] X \mathrm{~F}_{2}$ for neutronscattering measurements from samples with different AF film compositions. The AF domain size $\Delta_{(100)}$ was obtained from the measured width $\Sigma$ (rms) of the (100) AF Bragg reflections (Fig. 7). For these measurements $2 \theta=88^{\circ}$ and $\delta d=0.047 \AA$. $H_{C}$ is the coercive field.

\begin{tabular}{lcccccc}
\hline \hline \multicolumn{1}{c}{ Sample } & $\begin{array}{c}H_{E} \\
(\mathrm{Oe})\end{array}$ & $\begin{array}{c}H_{C} \\
(\mathrm{Oe})\end{array}$ & $\begin{array}{c}\left|J_{\text {int }}\right| \\
\left(\mathrm{erg} / \mathrm{cm}^{2}\right)\end{array}$ & $\begin{array}{c}\Delta_{100} \\
(\AA)\end{array}$ & $\begin{array}{c}\Sigma \\
(\AA)\end{array}$ & $\begin{array}{c}\text { AF film thickness } \\
(\AA)\end{array}$ \\
\hline $\mathrm{Co} / \mathrm{Ni}_{0.55} \mathrm{Fe}_{0.45} \mathrm{~F}_{2}$ & +466 & 217 & 1.7 & $894 \pm 376$ & $0.052 \pm 0.004$ & 450 \\
$\mathrm{Co} / \mathrm{Zn}_{0.30} \mathrm{Fe}_{0.70} \mathrm{~F}_{2}$ & -234 & 45 & 0.6 & $419 \pm 85$ & $0.058 \pm 0.004$ & 610 \\
$\mathrm{Co} / \mathrm{FeF}_{2}$ & \pm 1750 & 1000 & 1.2 & $343 \pm 42$ & $0.061 \pm 0.003$ & 400 \\
\hline \hline
\end{tabular}


$\Delta_{\mathrm{xtal}}=310 \AA$; thus, if an AF domain spans the entire thickness of the AF layer, then the lateral crystal domain size may constrain the AF domain size to be smaller than the film thickness.

An alternative explanation for the small AF domain size is that an AF domain does not span the entire film thickness. For example, AF domains may be stacked one on another. Support for this explanation was given in Ref. 21, where the magnetic structure of the FM/AF interfacial region was shown to be distinct from the AF film bulk with a thickness of $\sim 50 \AA$. Our neutron diffraction study is not very sensitive to the AF structure of the interfacial region.

From the apparent insensitivity of $H_{E}$ to the AF domain size of the film bulk, one might conclude that the spin structure of the AF film bulk is not relevant to exchange bias. However, this conclusion runs counter to four experimental observations that suggest the AF film bulk is important to $H_{E}$ : (1) The AF film thickness must exceed a critical dimension $(\sim 100 \AA)$ in order to support exchange bias. ${ }^{81,82}$ (2) The pinned magnetization in the AF bulk is an anchor for the unidirectional anisotropy giving rise to exchange bias. ${ }^{32}$ (3) The net uncompensated magnetization in the AF film bulk and positive exchange bias are correlated. ${ }^{21}$ (4) The exchange bias in FM/AF/FM trilayers is strongly dependent on the relative magnetic state of the two FM's; thus, implying a strong coupling across the thick AF. ${ }^{83}$

A different view-one wherein the AF bulk spin structure does influence $H_{E}$-is that the AF domain state of the film bulk propagates to the FM/AF interface affecting the local domain state. For example, the size of the AF domains in the bulk may determine how many uncompensated magnetic spins are available (in the bulk and at the FM/AF interface) for aligning and pinning by the cooling field to produce $H_{E}{ }^{8,10}$ The size of the AF domains relative to the FM domains can determine the temperature dependence of exchange bias (averaging vs nonaveraging). ${ }^{25-27}$ Furthermore, the AF film bulk also plays a role in determining the sign of $H_{E}$. When the product of the net uncompensated magnetization in the AF film bulk and the cooling field become so large that antiferromagnetic exchange coupling across the FM/AF interface is frustrated, $H_{E}$ will be positive. ${ }^{21}$

We also compared the AF domain sizes for films with different compositions. ${ }^{84}$ Here again, these dimensions were generally smaller than the thickness of the AF films and reasonably similar despite the very different values of $H_{E}$.

The AF domains in our fluoride system $(\sim 300 \AA)$ are about 33 times smaller than the AF regions imaged in $\mathrm{Co} / \mathrm{LaFeO}_{3}(\sim 1 \mu \mathrm{m})$ with PEEM. ${ }^{28}$ It should be noted that PEEM may not be able to image 300- $\AA$-sized AF domains, so the observation of $1-\mu \mathrm{m}$-sized $\mathrm{AF}$ region does not rule out smaller domains, or that larger regions of uncompensated magnetization are comprised of smaller AF domains. We measured the crystal domain size for the $\mathrm{LaFeO}_{3}$ film with GIXD and obtained a lower bound of $\Delta_{\text {xtal }}\left(\mathrm{LaFeO}_{3}\right)$ $>0.8 \mu \mathrm{m}$ [Fig. 4(d)]. $\Delta_{\text {xtal }}\left(\mathrm{LaFeO}_{3}\right)$ is again similar to the micrometer-sized AF regions imaged with PEEM, suggesting that the crystal domain size constrains the AF domains.

There is a remarkable scaling between the interface coupling constant (a measure of interfacial coupling strength giving rise to unidirectional anisotropy and $H_{E}$ ) and the $\mathrm{AF}$ domain sizes for the $\mathrm{Co} / X \mathrm{~F}_{2}$ and $\mathrm{Co} / \mathrm{LaFeO}_{3}$ systems. The interface coupling constant. ${ }^{4} J_{\text {int }}=H_{E} M_{\mathrm{Co}} t_{\mathrm{Co}}$, where $M_{\mathrm{Co}}$ and $t_{\mathrm{Co}}$ are the magnetization and thickness of the Co layers, is about 100 times larger for our fluoride samples compared to that for the $\mathrm{Co} / \mathrm{LaFeO}_{3}$ sample. ${ }^{34}$ Thus, while the AF domain sizes remain comparable in the fluoride systems regardless of exchange bias (the difference between $H_{E}$ for different alloys is mostly explained by differences in the thickness of the $\mathrm{Co}$ layers), a small AF domain size may be a prerequisite for producing large numbers of uncompensated spins that can be aligned and pinned to promote large $J_{\text {int }}$ and, hence, large $\left|H_{E}\right|$ observed in the AF fluorides (compared to $\mathrm{LaFeO}_{3}$ ).

The fact that the AF domain sizes are comparable for large $\pm\left|H_{E}\right|$ (see Table I) is not surprising since recent studies $^{21,27}$ suggest that $H_{E}$ is related to the work done to create a domain wall in the ferromagnet, and the work done is independent of whether the FM/AF interface is programed (through field cooling) to have positive or negative exchange bias. Thus, the sign of $H_{E}$ might not be influenced by the AF domain size, but is influenced by the direction of the uncompensated magnetization in the $\mathrm{AF}^{21}$

\section{B. Comparison of the Néel and blocking temperatures}

A second result of our experiments involves comparison of the Néel $\left(T_{N}=55 \pm 2 \mathrm{~K}\right)$ and blocking $\left(T_{B}\right.$ $=52.2 \pm 0.3 \mathrm{~K})$ temperatures for the $\mathrm{Co} / \mathrm{Zn}_{0.30} \mathrm{Fe}_{0.70} \mathrm{~F}_{2}$ sample. ${ }^{85}$ The higher value of $T_{N}$ compared to $T_{B}$ is consistent with the higher value of $T_{N}$ deduced from the pinned magnetization measured in samples with higher concentrations of $\mathrm{Zn} .{ }^{16}$ For high concentrations of $\mathrm{Zn}$, suppression of $T_{B}$ compared to $T_{N}$ may result from a large number of nonmagnetic defects which make AF domain reversal possible near $T_{N}$ when the ferromagnetic layer is reversed.

\section{CONCLUSIONS}

In conclusion, we have measured a characteristic dimension of antiferromagnetic domains in three samples with different chemical compositions and exchange bias and in one sample with a variable exchange bias (controlled with temperature and orientation of the cooling field). The AF domain sizes were consistently similar and small. For one sample it was possible to obtain both the AF and the lateral crystal domain sizes, and we found these sizes were also similarsuggesting that imperfections of the AF crystal structure may constrain the AF domain size.

We conclude that the AF domain size of the film bulk does not directly influence the magnitude or sign of exchange bias. ${ }^{86}$ Rather, we suggest that the size of the AF domains in the bulk influences the local (interfacial) AF domain state. The interactions between the cooling field, FM domains, and uncompensated spins near the FM/AF interface and AF film bulk ultimately determine the magnitude and sign of exchange bias. In the case of the fluoride systems, crystallographic imperfections of the atomic lattice, e.g., low angle grain boundaries in the untwinned single crystalline films, or twins in epitaxial films grown on $\mathrm{MgO},{ }^{87}$ may constrain the size of the AF domains to be small. Small AF domains may be accompanied by large numbers of uncom- 
pensated spins ${ }^{21,32}$ that can be aligned and pinned to produce strong interface coupling promoting large exchange bias. On the other hand, the AF regions in the $\mathrm{Co} / \mathrm{LaFeO}_{3}$ system are large and, consequently, the number of uncompensated spins might be relatively small ${ }^{34}$ compared to $\mathrm{Co} / \mathrm{FeF}_{2}$, yielding small exchange bias. We suggest small AF domains in the film bulk are a prerequisite, but not a sufficient condition, for large exchange bias.

We also measured the temperature dependencies of exchange bias and the magnetic ordering of the $\mathrm{Co} / \mathrm{Zn}_{0.30} \mathrm{Fe}_{0.70} \mathrm{~F}_{2}$ sample and found the blocking temperature for exchange bias was $3 \mathrm{~K}$ below the Néel temperature of the AF film.

Finally, we demonstrated that by using time-of-flight neutron scattering, the magnetic structure of a thin antiferromagnetic film can be trivially characterized. Further, data from single crystal films with masses $<10 \mu \mathrm{g}$ (or thicknesses of hundreds of angstroms) are possible to obtain in as few as 2 h. With the advent of more powerful pulsed-spallation sources, growth of somewhat larger samples and a bit of patience, comparable data using the techniques demonstrated here should be possible to collect from 10 - $\AA$-thick filmsones that might yield insight into the magnetic structure of the FM/AF interface.

\section{ACKNOWLEDGMENTS}

This work was supported by the Office of Basic Energy Science, U.S. Department of Energy, BES-DMS at LANSCE and UCSD, and the National Science Foundation (Grant No. DMR-0400578) at WVU. This work benefited from the use of the Lujan Neutron Scattering Center at LANSCE, which is funded by the Department of Energy's Office of Basic Energy Science. Los Alamos National Laboratory is operated by Los Alamos National Security LLC under DOE Contract No. DE-AC52-06NA25396. We acknowledge valuable discussions with R. Pynn (IU).
* Present address: Department of Physics and Astronomy, Sonoma State University, 1801 East Cotati Ave., Rohnert Park, CA 94928, USA.

${ }^{1}$ W. H. Meiklejohn and C. P. Bean, Phys. Rev. 102, 1413 (1956).

${ }^{2}$ W. H. Meiklejohn and C. P. Bean, Phys. Rev. 105, 904 (1957).

${ }^{3}$ J. Nogués and I. K. Schuller, J. Magn. Magn. Mater. 192, 203 (1999).

${ }^{4}$ A. E. Berkowitz and K. Takano, J. Magn. Magn. Mater. 200, 552 (1999).

${ }^{5}$ B. Dieny, J. Magn. Magn. Mater. 136, 335 (1994).

${ }^{6}$ R. L. Stamps, J. Phys. D 33, R247 (2000).

${ }^{7}$ M. Kiwi, J. Magn. Magn. Mater. 234, 584 (2001).

${ }^{8}$ A. P. Malozemoff, Phys. Rev. B 35, 3679 (1987).

${ }^{9}$ D. Mauri, H. C. Siegmann, and P. S. Bagus, J. Appl. Phys. 62, 3047 (1987)

${ }^{10}$ T. C. Schulthess and W. H. Butler, Phys. Rev. Lett. 81, 4516 (1998).

${ }^{11}$ P. Miltényi, M. Gierlings, J. Keller, B. Beschoten, G. Güntherodt, U. Nowak, and K. D. Usadel, Phys. Rev. Lett. 84, 4224 (2000).

${ }^{12}$ U. Nowak, K. D. Usadel, J. Keller, P. Miltényi, B. Beschoten, and G. Güntherodt, Phys. Rev. B 66, 014430 (2002).

${ }^{13}$ J. Keller, P. Miltényi, B. Beschoten, G. Güntherodt, U. Nowak, and K. D. Usadel, Phys. Rev. B 66, 014431 (2002).

${ }^{14}$ J.-I. Hong, T. Leo, D. J. Smith, and A. E. Berkowitz, Phys. Rev. Lett. 96, 117204 (2006).

${ }^{15}$ H. Shi, D. Lederman, and E. E. Fullerton, J. Appl. Phys. 91, 7763 (2002).

${ }^{16}$ H. Shi, Z. Liu, and D. Lederman, Phys. Rev. B 72, 224417 (2005).

${ }^{17}$ M. Grimsditch, A. Hoffmann, P. Vavassori, H. Shi, and D. Lederman, Phys. Rev. Lett. 90, 257201 (2003).

${ }^{18}$ C. Leighton, H. Suhl, M. J. Pechan, R. Compton, J. Nogués, and I. K. Schuller, J. Appl. Phys. 92, 1483 (2002).

${ }^{19}$ G. J. Mata, E. Pestana, M. Kiwi, and H. Dreysse, Phys. Rev. B 74, 144407 (2006).

${ }^{20}$ J. Nogués, D. Lederman, T. J. Moran, and I. K. Schuller, Phys. Rev. Lett. 76, 4624 (1996).

${ }^{21}$ M. R. Fitzsimmons, B. J. Kirby, S. Roy, Zhi-Pan Li, Igor V.
Roshchin, S. K. Sinha, and Ivan K. Schuller, Phys. Rev. B 75, 214412 (2007).

${ }^{22}$ M. Cheon, Z. Liu, and D. Lederman, Appl. Phys. Lett. 90, 012511 (2007).

${ }^{23}$ F. Radu, A. Westphalen, K. Theis-Bröhl, and H. Zabel, J. Phys.: Condens. Matter 18, L29 (2006).

${ }^{24}$ V. K. Valev, M. Gruyters, A. Kirilyuk, and Th. Rasing, Phys. Rev. Lett. 96, 067206 (2006).

${ }^{25}$ I. V. Roshchin, O. Petracic, R. Morales, Z.-P. Li, X. Batlle, and I. K. Schuller, Europhys. Lett. 71, 297 (2005).

${ }^{26}$ O. Petracic, Z.-P. Li, I. V. Roshchin, M. Viret, R. Morales, X. Batlle, and I. K. Schuller, Appl. Phys. Lett. 87, 222509 (2005).

${ }^{27}$ Y. Henry, S. Mangin, T. Hauet, and F. Montaigne, Phys. Rev. B 73, 134420 (2006).

${ }^{28}$ F. Nolting, A. Scholl, J. Stöhr, J. W. Seo, J. Fompeyrine, H. Siegwart, J.-P. Locquet, S. Anders, J. Lüning, E. E. Fullerton, M. F. Toney, M. R. Scheinfein, and H. A. Padmore, Nature (London) 405, 767 (2000).

${ }^{29}$ A. Scholl, J. Stöhr, J. Lüning, J. W. Seo, J. Fompeyrine, H. Siegwart, J.-P. Locquet, F. Nolting, S. Anders, E. E. Fullerton, M. R. Scheinfein, and H. A. Padmore, Science 287, 1014 (2000).

${ }^{30}$ A. Scholl, F. Nolting, J. W. Seo, H. Ohldag, J. Stöhr, S. Raoux, J.-P. Locquet, and J. Fompeyrine, Appl. Phys. Lett. 85, 4085 (2004).

${ }^{31}$ H. Ohldag, H. Shi, E. Arenholz, J. Stöhr, and D. Lederman, Phys. Rev. Lett. 96, 027203 (2006).

${ }^{32}$ S. Roy, M. R. Fitzsimmons, S. Park, M. Dorn, O. Petracic, I. V. Roshchin, Z.-P. Li, X. Batlle, R. Morales, A. Misra, X. Zhang, K. Chesnel, J. B. Kortright, S. K. Sinha, and I. K. Schuller, Phys. Rev. Lett. 95, 047201 (2005).

${ }^{33}$ M. Gruyters and D. Schmitz, Phys. Rev. Lett. 100, 077205 (2008).

${ }^{34}$ A. Hoffmann, J. W. Seo, M. R. Fitzsimmons, H. Siegwart, J. Fompeyrine, J. P. Locquet, J. A. Dura, and C. F. Majkrzak, Phys. Rev. B 66, 220406(R) (2002).

${ }^{35}$ S. Mangin, F. Montaigne, and A. Schuhl, Phys. Rev. B 68, 140404(R) (2003).

${ }^{36}$ H. Shi and D. Lederman, Phys. Rev. B 66, 094426 (2002). 
${ }^{37}$ S. K. Sinha, E. B. Sirota, S. Garoff, and H. B. Stanley, Phys. Rev. B 38, 2297 (1988).

${ }^{38}$ R. Pynn, Phys. Rev. B 45, 602 (1992).

${ }^{39}$ M. R. Fitzsimmons and E. Burkel, Phys. Rev. B 47, 8436 (1993).

${ }^{40}$ L. G. Parratt, Phys. Rev. 95, 359 (1954).

${ }^{41}$ B. Vidal and P. Vincent, Appl. Opt. 23, 1794 (1984).

${ }^{42}$ M. R. Fitzsimmons and C. F. Majkrzak, in Modern Techniques for Characterizing Magnetic Materials, edited by Y. Zhu (Kluwer, Boston, 2005), pp. 107-152.

${ }^{43}$ W. H. Press, B. P. Flannery, S. A. Teukolsky, and W. T. Vetterling, Numerical Recipes, The Art of Scientific Computing (Cambridge University Press, Cambridge, 1986), p. 294.

${ }^{44}$ The normalized $\xi^{2}$ metrics were 21,7 , and 4 for the $\mathrm{Co} / \mathrm{Ni}_{0.55} \mathrm{Fe}_{0.45} \mathrm{~F}_{2}, \mathrm{Co} / \mathrm{Zn}_{0.30} \mathrm{Fe}_{0.70} \mathrm{~F}_{2}$, and $\mathrm{Co} / \mathrm{FeF}_{2}$ samples, respectively.

${ }^{45}$ H. Dosch, Phys. Rev. B 35, 2137 (1987).

${ }^{46} \mathrm{The} \mathrm{Al}$ and $\mathrm{Co}$ layers for the alloy samples were too thick for GIXD.

${ }^{47}$ M. A. Krivoglaz, Theory of X-ray and Thermal-Neutron Scattering by Real Crystals (Plenum, New York, 1969).

${ }^{48}$ Let $\sigma_{\mathrm{FeF}_{2}}$ be the measured width (rms) of a Bragg reflection from the $\mathrm{FeF}_{2}$ film. $\Gamma$ appearing in Fig. 4 is equal to $\sqrt{8 \ln 2}\left(\sigma_{\mathrm{FeF}_{2}}^{2}\right.$ $-\sigma_{\text {ins }}^{2}$.

${ }^{49}$ H. Lipson and H. Steeple, Interpretation of X-ray Powder Diffraction Patterns (St. Martin's, New York, 1970).

${ }^{50}$ The error on $\Gamma$ for the $\mathrm{FeF}_{2}$ reflections of $0.00135 \AA^{-1}$ is the standard deviation of the values of $\Gamma$ for the GaAs reflections.

${ }^{51}$ J. Narayan and B. C. Larson, J. Appl. Phys. 93, 278 (2003).

${ }^{52}$ D. B. Williams and C. B. Carter, Transmission Electron Microscopy (Plenum, New York, 1996), p. 447.

${ }^{53}$ L. B. Freund, MRS Bull. 17, 52 (1992).

${ }^{54}$ J. Mattsson, C. Djurberg, and P. Nordblad, J. Magn. Magn. Mater. 136, L23 (1994).

${ }^{55}$ M. Lederman, J. Hammann, and R. Orbach, Physica B (Amsterdam) 165-166, 179 (1990).

${ }^{56} \mathrm{Ch}$. Binek, Xi Chen, A. Hochstrat, and W. Kleemann, J. Magn. Magn. Mater. 240, 257 (2002).

${ }^{57}$ M. Ooi, T. Ino, G. Muhrer, E. J. Pitcher, G. J. Russell, P. D. Ferguson, E. B. Iverson, D. Freeman, and Y. Kiyanagi, Nucl. Instrum. Methods Phys. Res. A 566, 699 (2006).

${ }^{58}$ The divergence in degrees of the neutron beam from the guide is $0.23 \lambda$ for $\lambda$ in angstroms.

${ }^{59}$ The mosaic spreads of the samples were much less than the divergence of the neutron beam incident on the sample.

${ }^{60}$ The largest neutron beam on Asterix is $5 \times 5 \mathrm{~cm}^{2}$.

${ }^{61}$ See http://www.ncnr.nist.gov/instruments/bt $9 /$

${ }^{62}$ H. Shi, D. Lederman, K. V. O'Donovan, and J. A. Borchers, Phys. Rev. B 69, 214416 (2004).

${ }^{63}$ The relation is valid for small or Gaussian-distributed values of $d \lambda / \lambda$ and $d \theta \cot \theta$. Given the Gaussian profile of the (100) Bragg reflection, the relation is reasonable to use.

${ }^{64} \mathrm{~B}$. Fultz and J. Howe, Transmission Electron Microscopy and Diffractometry of Materials, 2nd ed. (Springer, Berlin, 2002), p. 433.

${ }^{65}$ M. R. Fitzsimmons, Nucl. Instrum. Methods Phys. Res. A 383, 549 (1996).

${ }^{66}$ The $\sigma_{m}$ typically increases with $\lambda$ below $6 \AA$ and then saturates.

${ }^{67}$ Note that the Bragg reflection from the graphite sample is highly asymmetric in Fig. 8-typical of an instrumental-resolution- limited intensity profile. In contrast, the Bragg reflection from the $\mathrm{Zn}_{0.30} \mathrm{Fe}_{0.70} \mathrm{~F}_{2}$ sample in Fig. 7 is broadened yielding a more symmetric intensity profile.

${ }^{68}$ This experiment allowed us to demonstrate the combination of inelastic scattering and reflectometry with an energy resolution of $30 \mu \mathrm{eV}$.

${ }^{69}$ C. G. Shull, W. A. Strauser, and E. O. Wollan, Phys. Rev. 83, 333 (1951).

${ }^{70}$ B. E. Warren, X-ray Diffraction (Dover, New York, 1990), p. 253.

${ }^{71}$ Since the (100) wave vector lies $45^{\circ}$ from the sample's surface normal, the dimension of the domains corresponds to this direction (i.e., a dimension that is neither purely perpendicular nor parallel to the sample's surface).

${ }^{72}$ M. R. Fitzsimmons, J. A. Eastman, M. Müller-Stach, and G. Wallner, Phys. Rev. B 44, 2452 (1991).

${ }^{73}$ The in-plane microstrain can not be measured with GIXD for either of the $\mathrm{Zn}_{0.30} \mathrm{Fe}_{0.70} \mathrm{~F}_{2}$ or the $\mathrm{Ni}_{0.55} \mathrm{Fe}_{0.45} \mathrm{~F}_{2}$ films because the $\mathrm{Co}$ and $\mathrm{Al}$ overlayers are so thick that the $\mathrm{x}$-ray beam is absorbed by the overlayers.

${ }^{74}$ The strain term is $25 \%$ of the domain-size term.

${ }^{75}$ N. W. Ashcroft and N. D. Mermin, Solid State Physics (Holt, Rinehart, and Winston, New York, 1976), p. 699.

${ }^{76}$ N. Rosov, A. Kleinhammes, P. Lidbjörk, C. Hohenemser, and M. Eibschütz, Phys. Rev. B 37, 3265 (1988).

${ }^{77}$ G. E. Bacon, Neutron Diffraction (Clarendon, Oxford, 1975).

${ }^{78}$ G. L. Squires, Introduction to the Theory of Thermal Neutron Scattering (Dover, New York, 1978), pp. 146-152.

${ }^{79}$ A. Tucciarone, H. Y. Lau, L. M. Corliss, A. Delapalme, and J. M. Hastings, Phys. Rev. B 4, 3206 (1971).

${ }^{80}$ D. Lederman, J. Nogués, and I. K. Schuller, Phys. Rev. B 56, 2332 (1997).

${ }^{81}$ K. Nishioka, S. Shigematsu, T. Imagawa, and S. Narishige, J. Appl. Phys. 83, 3233 (1998).

${ }^{82}$ Y. Ozaki, K. Shimoyama, S. Iwata, and S. Tsunashima, IEEE Trans. Magn. 36, 2888 (2000).

${ }^{83}$ R. Morales, Z.-P. Li, J. Olamit, Kai Liu, J. M. Alameda, and Ivan K. Schuller (unpublished).

${ }^{84}$ The observation of a (100) AF Bragg reflection for the $\mathrm{Co} / \mathrm{Ni}_{0.45} \mathrm{Fe}_{0.55} \mathrm{~F}_{2}$ sample means that the (100) planes have a net ferromagnetic moment that is not consistent with the AF structure of bulk $\mathrm{NiF}_{2}$.

${ }^{85}$ The difference between $T_{B}$ and $T_{N}$ is significant provided the thermometry used in the SQUID and neutron experiment performed similarly (both used calibrated Cernox sensors). A better approach would be to measure the temperature dependencies of the exchange bias with polarized neutron reflectometry and $T_{N}$ with neutron diffraction using the same thermometermeasurements planned for the future with Asterix.

${ }^{86}$ We note that in Refs. 25 and 26, it was concluded the size of the domain of uncompensated magnetization in the AF affected the sign and magnitude of exchange bias. This dimension is not the $\mathrm{AF}$ domain size we discuss in the present paper. The AF domain size refers to the size of the domain over which long range order of the AF spin lattice exists.

${ }^{87}$ M. R. Fitzsimmons, C. Leighton, J. Nogués, A. Hoffmann, K. Liu, C. F. Majkrzak, J. A. Dura, J. R. Groves, R. W. Springer, and P. N. Arendt, V. Leiner, H. Lauter, and I. K. Schuller, Phys. Rev. B 65, 134436 (2002). 